study is described, concerning the way in which colicins act on bacterial cells. These substances are proteins (isolated from so-called colicinogenic strains of $E$. coli) which have powerful antibiotic activity against sensitive strains. They are singular in being adsorbed into specific sites on the membranes of sensitive cells, but not penetrating into the cells. Such sites are only present in sensitive strains. Senior, Kwasniak and Holland have studied the colicin E3 (molecular weight $60,000)$ which inhibits protein synthesis. In cells exposed to E3, protein biosynthesis is arrested after about five minutes, and is paralleled by the appearance of polyribosomes which dissociate into ribosomal

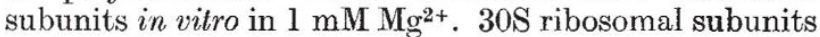
isolated from cells treated with E3 are correspondingly deficient in protein synthesis in vitro, and Senior et al. suggest that the lesion causing both the inhibition of in vivo protein synthesis and the curious instability of the polysomes is a modification of the $30 \mathrm{~S}$ subunits. They show that this can occur whether or not the $30 \mathrm{~S}$ subunits are actively engaged in protein synthesis. Further study of the mechanism whereby attachment of the colicin to the surface of a cell can cause such a modification of the ribosomal subunits will certainly be of great importance in explaining the means of communication between the cell membrane and the interior.

\section{X-RAY ASTRONOMY}

\section{Radiation and Discrete Sources}

\section{by our Cosmology Correspondent}

THE first of the new informal specialist discussion groups arranged by the Royal Astronomical Society to precede the regular monthly meetings was held on November 13. Topics for discussion were the isotropic diffuse background radiation, pulsar radiation and discrete X-ray sources. The meeting was an undoubted success, confirming the belief that small meetings of experts in one field are more profitable than larger meetings covering diverse topics each of which is understood only by a minority of the audience.

Dr M. Longair (University of Cambridge) gave his own interpretation of the shape of the spectrum of high energy background photons. This follows a power law with index roughly $\mathbf{1 . 5}$ for energies below $50 \mathrm{keV}$ and 2.2 for energies above $50 \mathrm{keV}$ but below $1 \mathrm{MeV}$. The few available data at very low and very high energies deviate from this simple behaviour, with an apparent excess of photons at each end of the scale. But the principal point of discussion concerned the reality of the "knee" at $50 \mathrm{keV}$. Some theoreticians have argued that this appears only because of observational difficultics, which arise because this change of slope occurs just in the energy range where the sort of detector used to study the radiation changes from proportional counters to scintillation counters.

It was generally agreed that this break is indeed real, and that the problem now confronting observers is to determine the exact range in energies over which the change in slope occurs. At present, the data are sufficiently inconclusive that almost any theory of the origin of this background radiation can be adjusted to allow for the change, but most will fall by the wayside if observations can be improved by a factor of 10 or more.

The isotropy of this radiation was also a point of discussion. Because an energy density of only $10^{-4} \mathrm{eV}$ $\mathrm{cm}^{-3}$ is required to produce the radiation, many models can also be constructed to explain this. It is now clear that the radiation really is isotropic to a very large degree, and that if it is produced by many discrete sources of equal intensity then more than $10^{4}$ sources per steradian are required. For this reason, models in which the radiation is produced uniformly throughout intergalactic space (for example, by bremsstrahlung radiation from a uniform hot gas) seem preferable to those which require the radiation to originate in discrete regions and become uniformly spread out during its passage to Earth. It is significant that the isotropy needed for a discrete source model is greater than the isotropy of the distribution of galaxies in space.

Discussion of high energy emission from pulsars was severely limited because only the Crab pulsar is known to emit optical and X-ray pulses. But a very significant experiment carried out by the Bristol group earlier this year has provided meaa sure of the X-ray pulses from this source (NP 0532) above $600 \mathrm{keV}$. Both the main pulse and precursor seen optically and at radio frequencies werc detected, but at these high energies the precursor becomes three times more powerful than the main pulse, which is itself stronger than at lower frequencies. The fraction of the total emission energy which is in the pulse is therefore very large, in contradiction to the earlier belief that only a small part of a pulsar's emission occurs in the pulses. The most profitable way to find other high energy pulsars is therefore to look at young supernova remnants in other galaxies.

Dr M. Rees (University of Cambridge) suggested that the energy content of the pulses during the first months of a pulsar's life might be enough to make them visible as far away as the Virgo Cluster, covering a volume of space in which an average of one supernova a year occurs. Unfortunately, for the first few months of its life a pulsar would be obscured by the cloud of gas expanding away from it as a result of the explosion in which it originated, so that it is not certain that the pulsar will still be strong enough to be seen when the obscuration clears. But high energy astronomers will certainly be looking at hopeful cases in future.

Most of the discussion of discrete X-ray sources centred on the archetypes of the two types of source best known, Sco X-1 and the Crab. Very little of this covered any new ground, although it does seem that there is a consensus in favour of accepting the marginal evidence for greatly broadened lines of highly ionized iron in the spectrum of Sco X-1. The next new developments in this part of the field will probably result from the studies just commencing of the new class of bright low energy discrete sources (with emission only below $\sim 2 \mathrm{keV}$ ) like the Cygnus loop. These should provide spectra in which many lines will stand out, and give the theoreticians another chance to revise their estimates of the relative abundances of the elements in our galaxy.

\section{EARTH'S HEAT}

\section{Equality over Land and Sea}

\section{from our Geomagnetism Correspondent}

THe equality of the average heat flows through continents and oceans is one of the major mysteries in geophysics. If, as has commonly been assumed, the principal contribution to the Earth's heat flux is from 